Die Schweiz und die humanitäre Aktion

\title{
Der Humanitäre Bereich In Der Aussenpolitik Der Schweiz
}

Jean F. Freymond

\section{(2) OpenEdition \\ 1 Journals}

Electronic version

URL: http://journals.openedition.org/sjep/628

DOI: 10.4000/sjep.628

ISSN: 1663-9677

Publisher

Institut de hautes études internationales et du développement

Printed version

Date of publication: 1 janvier 1999

Number of pages: $25-38$

ISSN: 1660-5926

\section{Electronic reference}

Jean F. Freymond, «Der Humanitäre Bereich In Der Aussenpolitik Der Schweiz », Schweizerisches Jahrbuch für Entwicklungspolitik [Online], 18| 1999, Online erschienen am: 18 Juli 2012, abgerufen am 08 September 2020. URL : http://journals.openedition.org/sjep/628 ; DOI : https://doi.org/10.4000/ sjep.628 


\title{
DER HUMANITÄRE BEREICH IN DER AUSSENPOLITIK DER SCHWEIZ
}

\author{
JEAN F. FREYMOND*
}

A

NFANG AUgust 1998 an den Ufern des Kongo, weit weg von der Bundeshauptstadt Bern, Tausende von Kilometern von den Orten entfernt, wo sich die Aussenpolitik der Schweiz entscheidet, hat der Wahnsinn der Menschen zugeschlagen und ein Gefolge von Verwüstung und Greueln hinterlassen, Früchte eines Hasses, den man erweckt hat: zerstörte Stadtviertel und Infrastruktureinrichtungen, unschuldige oder schuldige Menschen, die verfolgt werden und sich verborgen halten, um dem schrecklichen Tod zu entgehen, dem jene, die nicht soviel Glück hatten, zum Opfer gefallen sind. Und seither arbeiten Männer und Frauen, um dem Leben all derer, die sich auf dem Durchzug des Sturms befanden, wieder etwas Menschlichkeit zu verleihen. Unter diesen Männern und Frauen von fast überallher sind einige, Vertreter der Schweizer Regierung, Diplomaten, Mitarbeiter der Direktion für Entwicklung und Zusammenarbeit (DEZA) und Delegierte des Internationalen Komitees vom Roten Kreuz (IKRK), die nachdem sie zunächst dazu beigetragen hatten, dass viele der Personen, die sich versteckt hielten, weggehen konnten, sodann einem ganzen Stadtviertel wieder Hoffnung einzuflössen versuchten, indem sie unter anderem beim Wiederaufbau eines Krankenhauses mithalfen. Dieses ausgewählte Beispiel soll gleich zu Anfang darauf hinweisen, dass die Stellung des humanitären Bereichs in der Aussenpolitik der Schweiz mehrere Gesichter hat, dass der humanitäre Bereich vor Ort in Erscheinung tritt und dass er sich letztlich dort vor allem konkretisieren muss.

Die Stellung des humanitären Bereichs ist heutzutage bedeutend. Der humanitäre Bereich begann sie sozusagen seit der Gründung des Bundesstaates 1848 einzunehmen, genauer gesagt, seit der Gründung des Internationalen Komitees für die Verwundetenpflege 1863 und der Einladung des Bundesrates an die europäischen Staaten, die Vereinigten Staaten, Brasilien und Mexiko, am Kongress teilzunehmen, der am 22. August 1864 mit der Annahme der ersten Genfer Konvention zur Verbesserung des Loses der Verwundeten und Kranken der Streitkräfte im Felde abschloss.

Die damalige Aussenpolitik der Schweiz umfasst zwei wesentliche Komponenten: «eine Aussenpolitik im engeren Sinne, beschränkt, von grosser Zurückhaltung geprägt, ohne Kühnheit $»^{2}$, deren Hauptfunktion darin liegt, die Unabhängigkeit und die Neutralität des Landes zu wahren. Kann es anders sein bei einem zusammengewürfelten, zudem neutralen Kleinstaat mit zerbrechlicher Identität, dessen kürzliche Gründung auf Revolutionen und einen Bürgerkrieg

* Direktor, Centre for Applied Studies in International Negotiations, Genf. Der Verfasser möchte hier Gudrun Reiner danken, die einen Teil der mit dieser Studie verbundenen Forschung übernahm.

1. Name, den sich das Organ 1863 gibt, das später zum Internationalen Komitee vom Roten Kreuz wird.

2. Jean F. Freymond, «Réflexions sur le champ des relations extérieures de l'Etat et la complexité du système international", Annuaire suisse de science politique 28/1988, p. 229. 
folgt? Diesem Land fehlt es jedoch nicht an Mut, wenn es sein muss. Zeugen hierfür sind der Neuenburger Konflikt 1856, dessen Ziel es ist, vom König von Preussen die volle Unabhängigkeit des Kantons Neuenburg zu erlangen, oder auch die Asyl- und Flüchtlingspolitik. Zweite Komponente, «eine dynamische Sachpolitik, die vor allem zum Ziel hat, die Integration des Landes als lebensfähige Einheit im Rahmen eines europäischen und weltweiten Systems zu ermöglichen, das immer interdependenter und komplexer wird $»^{3}$. Diese Politik ist oft multilateral. Sie konkretisiert sich durch eine aktive Teilnahme der Eidgenossenschaft an den grossen internationalen technischen Konferenzen und durch ihren Beitritt zu den Verwaltungseinheiten, die den Keim der internationalen Organisationen des zwanzigsten Jahrhunderts bilden, von ihrer Gründung $\mathrm{an}^{4}$.

$\mathrm{Zu}$ diesen beiden Komponenten muss eine dritte hinzugefügt werden, die anderer Art und unterschiedlich ist: eine «humanitäre» oder Solidaritätspolitik, die in den Augen einiger das Ergebnis einer der Schweiz übertragenen «Mission» ist, durch welche sie sich auszeichnet und die ihr eine der Dimensionen ihrer Identität verleiht ${ }^{5}$. Im 19. Jahrhundert und fast bis Mitte des 20. Jahrhunderts ist diese Politik, von der man in den Worten vieler Bundesräte einen Widerhall fin$\operatorname{det}^{6}$, zuerst in der Rede anzutreffen. Sie drückt sich kaum durch ein systematisches Engagement des Staates in Form von humanitären Aktionen oder allgemeiner durch ein konkretes Interesse für die humanitäre Sache aus. So ist die Gründung des Roten Kreuzes vor allem das Werk des Genfer Komitees, dessen fünf Mitglieder, an der Spitze Henry Dunant, sich abmühen, reisen, diskutieren und überzeugen. Besonders auf Paris wird Druck ausgeübt. Napoleon III. unterstützt schliesslich die Idee einer diplomatischen Konferenz, die in Genf abgehalten werden soll, wobei er aber hinzufügt, dass sie vom Bundesrat einberufen werden sollte, um den diplomatischen Gepflogenheiten zu entsprechen. Erst dann begibt sich der Präsident des Genfer Komitees, Gustave Moynier, nach Bern, wo er sich mit den Bundesräten Jakob Dubs und Constant Fornerod trifft und um Unterstützung bittet, die ihm auch ohne Schwierigkeit gewährt wird? Auf diese Zeit geht die Rolle zurück, welche die Eidgenossenschaft seither bei der Entwicklung des humanitären Völkerrechts unaufhörlich gespielt hat, eine Rolle, deren treibende Kraft das Genfer Komitee war und weiterhin ist, dem Bern wiederholte und wachsende Unterstützung gewähren wird. Diese Unterstützung drückt sich durch die Einberufung weiterer diplomatischer Konferenzen in den Jahren 1906 und 1929 auf Anregung des Komitees aus, aus denen die Genfer Konventionen von 1906 und 1929 hervorgehen $^{8}$.

Gewiss engagiert sich die Eidgenossenschaft aus eigenem Anstoss im humanitären Bereich, so bei der Internierung der Armee des Generals Bourbaki im Deutsch-Französischen Krieg von 1870-71, ferner als sie im Ersten Weltkrieg

3. Ibid.

4. Ibid. und Jean F. Freymond, «La politique extérieure multilatérale de la Suisse: aspects structurels». Relations internationales, $\mathrm{n}^{\circ} 32$, hiver 1982 , pp. 463-477.

5. Jean F. Freymond, «La politique extérieure multilatérale...».

6. Vgl. Daniel Frei, Neutralität - Ideal oder Kalkül? Zweihundert Jahre aussenpolitisches Denken in der Schweiz. Frauenfeld, Verlag Huber, 1967.

7. Pierre Boissier, Histoire du Comité International de la Croix-Rouge. De Solferino à Tsoushima. Paris, Plon, 1963. Vgl. auch Documents Diplomatiques suisses 1848-1945. Band I (24.11.1848-30.11.1865) Bern, Benteli Verlag, 1990.

8. Vgl. Pierre Boissier, Histoire du Comité International de la Croix-Rouge, und André Durand, Histoire du Comité International de la Croix-Rouge. De Sarajevo à Hiroshima. Genève, Institut Henry-Dunant, 1978. 
schwerverletzte Kämpfer und im Zweiten Weltkrieg Zehntausende von Kindern auf ihrem Gebiet aufnimmt. Aber bis spät ins 20. Jahrhundert sind die internationalen humanitären Einsätze der Eidgenossenschaft vor allem sporadisch. Sie werden erst Ende des Zweiten Weltkriegs systematisch, und zwar bei der Gründung der Schweizer Spende zugunsten der Kriegsopfer 1944, dies auf Initiative der Eidgenossenschaft ${ }^{9}$. Die Einführung der Schweizer Spende geht vor allem auf den Willen zurück, sich durch eine grosszügige und uneigennützige gemeinschaftliche Anstrengung, die das Schweizer Volk mit dem Staat verbindet, am Wiederaufbau Europas zu beteiligen. Doch sie erfolgt auch im Rahmen einer noch während des Kriegs durchgeführten Reflexion über die schweizerische Nachkriegspolitik und die Grundsätze, auf denen diese Politik beruhen sollte. Die Wiedereingliederung einer isolierten Schweiz, deren Neutralität in Verruf gebracht und falsch verstanden wird, in die Gemeinschaft der Siegernationen findet nicht unter den besten Vorzeichen statt. In den Augen zahlreicher Kritiker hätte sich die internationale Solidarität durch eine Teilnahme der Schweiz am Krieg ausdrücken sollen. Auf diesen Vorwurf erwidert der Bundesrat - und dies ist das Verdienst des Vorstehers des damaligen eidgenössischen Politischen Departements (EPD), Max Petitpierre - indem er dieser Solidarität, die er eng mit der Neutralität verbindet, einen anderen Sinn gibt - wobei die eine in vieler Hinsicht nicht ohne die andere einhergeht. «Die wesentliche mit der Neutralität verbundene Pflicht», erklärt Max Petitpierre, «ist die der Solidarität. Sie muss sich, je nach den Umständen, auf zwei Arten erfüllen. In Kriegs-zeiten, den einzigen Zeiten, wo die Neutralität ihre Existenzberechtigung hat und ihre Wirkungen entfaltet, muss die Solidarität vor allem im humanitären Bereich zum Ausdruck kommen. Sie muss gewissermassen blind sein - in gleicher Weise wie die Justiz - und darf kein anderes Ziel haben als das, zu lindern, zu helfen, zu mindern. Sie ist die notwendige Folge eines Privilegs: jenes, verschont zu sein.

In Friedenszeiten oder in diesen unsicheren Zeiten wie derjenigen, in der wir heute leben, die keine Kriegszeiten sind, aber ohne dass dabei Friede herrscht, hat die Solidarität einen ganz anderen Charakter. Sie setzt die Zusammenarbeit mit den anderen Ländern voraus, um durch eine gemeinsame Anstrengung dasselbe Ziel zu erreichen. Diese Solidarität ist kein edles Gefühl: sie ist die Folge einer Interdependenz zwischen den Völkern; sie ist auch die Anerkennung einer Verantwortung. Sie wird zugunsten eines Allgemeininteresses ausgeübt, an dem jeder Staat teilhat, der diese Solidarität anerkennt $»^{10}$.

Die Nachkriegszeit stellt somit eine Wende in der humanitären Politik der Schweiz dar. Von der gelegentlichen Politik, die sie war, wird sie zur regelmässigen Politik. Von 1946 an bewilligen die eidgenössischen Räte wiederholt Kredite für humanitäre Hilfe und ausserdem eine substantielle Unterstützung für das IKRK. Diese Unterstützung erfolgt in Form von Beiträgen, die im Durchschnitt die Hälfte des Sitzbudgets der Institution - deren Ursprung auf 1931 zurückgeht - decken, und ausserordentlichen Beiträgen, die für spezifische Aktionen bestimmt sind. Die humanitäre Politik wird auf diese Weise nach und

9. Vgl. Documents Diplomatiques suisses 1848-1945. Band 15 (8.9.1943-8.5.1945) Bern, Benteli Verlag, 1992.

10. Max Petitpierre, «Allocution prononcée à Berne à un dîner de l'Association de la presse étrangère» le 23 novembre 1950, Max Petitpierre. Seize ans de neutralité active. Aspects de la politique étrangère de la Suisse (1945-1961). Neuchâtel, La Baconnière, 1980, p. 254. 
nach aufgebaut. Die Einsätze mehren sich. Sie nehmen manchmal bilateralen Charakter und manchmal die Form einer Unterstützung an die internationalen Organisationen mit humanitärem Zweck an, deren Gründungen seit 1946 aufeinanderfolgen ${ }^{11}$.

Weitere wichtige Wendepunkte sind die Gründung des Schweizerischen Katastrophenhilfekorps (SKH), die auf 1973 zurückgeht, und vor allem die Verabschiedung des Bundesgesetzes über die internationale Entwicklungszusammenarbeit und humanitäre Hilfe im Jahr 1976. Von nun an wird die humanitäre Politik der Schweiz die Form annehmen, die sie heutzutage hat.

$\triangle$ M ENDE DES 20. Jahrhunderts nimmt die humanitäre Hilfe immer noch ihren APlatz als «Eckpfeiler der schweizerischen Aussenpolitik» ein ${ }^{12}$. Sie will bewusst Ausdruck einer langen spezifischen Tradition sein, die oft, aber nicht ausschliesslich mit dem Rotkreuzgedanken verbunden wird. Sie ist weniger als früher die notwendige Folge einer Neutralität, «bei der davon ausgegangen wird, dass sie besondere Pflichten im humanitären Bereich mit sich bringt $\gg^{13}$ und die zum Bestreben führen muss, die Leiden zu lindern, von denen man selbst verschont geblieben ist. Die humanitäre Hilfe ist schliesslich und vor allem Ausdruck dieser internationalen Solidarität, die eines der vier Grundprinzipien darstellt, auf denen die Aussenpolitik der Eidgenossenschaft seit Ende des Zweiten Weltkriegs ausdrücklich beruht ${ }^{14}$. Zunächst eine Solidarität mit den Opfern von Naturkatastrophen oder den von Menschen verursachten Katastrophen - wo diese Opfer sich auch immer in der Welt befinden und welches ihre Nationalität, ihre Rasse, ihre Religion oder ihre Meinungen sind - Solidarität, deren Speerspitze die humanitäre Hilfe ist. Aber eine Solidarität, deren Bereich umfassender ist, da die humanitäre Politik im weiteren Sinne danach strebt, «den Menschen, seine Existenz, seine Würde und Freiheit zu schützen $»^{15}$.

Ein breit angelegtes Programm, das auf Anhieb die Frage der Definition dieser humanitären Politik im weiteren Sinne aufwirft. Der Bundesrat legt in seiner Botschaft über die Weiterführung der internationalen humanitären Hilfe der Eidgenossenschaft vom 25. Mai 1988 fest, was sie einschliesst: die Förderung und den Schutz der Menschenrechte, die Weiterentwicklung des humanitären Völkerrechts, die Unterstützung des IKRK, die internationale Flüchtlingspolitik, die Internierung von Kriegsgefangenen und die humanitäre Hilfe ${ }^{16}$. Er wählt somit einen beschränkenden und sektoriellen Ansatz, dessen Hauptbestandteile die humanitäre Hilfe, die Unterstützung des IKRK, die Weiterentwicklung des humanitären Völkerrechts und die Menschenrechtspolitik sind. Dieser Ansatz schliesst unter anderem die Entwicklungszusammenarbeit und die Asylpolitik - nicht aber

11. Botschaft des Bundesrates an die Bundesversammlung betreffend ein Bundesgesetz über die internationale Entwicklungszusammenarbeit und humanitäre Hilfe vom 19. März 1973, BBl 125, 1973, I, S. 883-886.

12. Botschaft des Bundesrates über die Weiterführung der internationalen humanitären Hilfe der Eidgenossenschaft vom 20. November 1996, S.3.

13. Pierre Micheli, «Les grandes lignes de la politique étrangère conduite par M. Max Petitpierre de 1945 à 1961 », Max Petitpierre. Seize ans de neutralité active, p. 33.

14. Joseph Boesch, «Epochen der Schweizerischen Aussenpolitik», Handbuch der schweizerischen Aussenpolitik, Bern, Paul Haupt, 1975, S. 120.

15. Botschaft des Bundesrates über die Weiterführung der internationalen humanitären Hilfe der Eidgenossenschaft vom 25. Mai 1988, BBI 140, 1988, II, S. 1213.

16. Ibid. 
die internationale Flüchtlingspolitik - aus der humanitären Politik aus. Mit anderen Worten stellt die humanitäre Politik im engeren Sinne nur einen Bereich der Solidaritätspolitik des Bundes dar neben anderen Bereichen, wie beispielsweise die Entwicklungszusammenarbeit oder die Asylpolitik. Diese humanitäre Politik hat zum gemeinsamen Nenner, dass sie zu allererst in Krisenzeiten gültig ist, oder während eine Krise droht, dass sie anstrebt, die Not der Menschen zu verhüten und unverzüglich zu lindern, und schliesslich, dass sie die praktische Umsetzung von humanitären Werten und Regeln fördern will.

Die Botschaft des Bundesrates betreffend ein Gesetz über die internationale Entwicklungszusammenarbeit und humanitäre Hilfe vom 19. März 1973 ist in dieser Beziehung explizit: "Humanitäre Hilfe und Entwicklungszusammenarbeit stellen miteinander insofern ein Ganzes dar, als sie als verschiedene Aspekte desselben Solidaritätselementes unserer Aussenpolitik zusammengehören ${ }^{17}$. Sie «haben ein gemeinsames Fundament in der mitmenschlichen Verpflichtung, Not, Elend und Armut anderer nicht auf sich beruhen zu lassen, sondern zu ihrer Überwindung das Mögliche beizutragen ${ }^{18}$. Und der Bundesrat fährt fort, indem er andere gemeinsame Züge hervorhebt, wie die enge Beziehung zwischen der humanitären Hilfe und der Entwicklungszusammenarbeit, von der er sagt, dass sie «auf den Leistungen der humanitären Hilfe aufbauen und sie fortführen» kann ${ }^{19}$.

Aber diese gemeinsamen Merkmale können nicht die klaren Unterschiede vergessen lassen, welche die technische Zusammenarbeit und die humanitäre Hilfe voneinander trennen. Zunächst die Tatsache, dass «die humanitäre Hilfe der Not und Gefahr innerhalb gegebener wirtschaftlicher und sozialer Verhältnisse direkt $\mathrm{zu}$ begegnen sucht $»^{20}$, wohingegen es «das Ziel der Entwicklungszusammenarbeit ist, zur Veränderung solcher Verhältnisse im Hinblick auf eine dauerhafte Verbesserung der Lebensbedingungen beizutragen und damit auch die Not und die Lebensgefährdung an ihren Wurzeln zu bekämpfen $»^{21}$. Diese tiefe und plötzliche Not kennt keine Grenzen. Die humanitäre Aktion hat daher Universalcharakter und kann prinzipiell sowohl die Länder des Südens als auch die Industrieländer betreffen. Die Entwicklungszusammenarbeit ist ihrerseits selektiv. Sie bezieht sich nur auf die Entwicklungsländer und auch nur auf einige unter ihnen.

Der Gesetzgeber behandelt die internationale Entwicklungszusammenarbeit und humanitäre Hilfe in ein und demselben Gesetz. Aber er stellt sorgfältig heraus, was sie voneinander unterscheidet. Dies widerspiegelt sich in den beiden Definitionen, die er annimmt :

«Die Entwicklungszusammenarbeit unterstützt die Entwicklungsländer im Bestreben, die Lebensbedingungen ihrer Bevölkerung zu verbessern. Sie soll dazu beitragen, dass diese Länder ihre Entwicklung aus eigener Kraft vorantreiben. Langfristig erstrebt sie besser ausgewogene Verhältnisse in der Völkergemeinschaft $»^{22}$.

17. Botschaft des Bundesrates an die Bundesversammlung betreffend ein Gesetz über die internationale Entwicklungszusammenarbeit und humanitäre Hilfe vom 19. März 1973, BBI 125, 1973, I, S. 873.

18. Ibid.

19. Ibid., S. 874 .

20. Ibid.

21. Ibid.

22. Bundesgesetz über die internationale Entwicklungszusammenarbeit und humanitäre Hilfe vom 19. März 1976. 
«Die humanitäre Hilfe soll mit Vorbeugungs- und Nothilfemassnahmen zur Erhaltung gefährdeten menschlichen Lebens sowie zur Linderung von Leiden beitragen; sie ist namentlich für die von Naturkatastrophen oder bewaffneten Konflikten heimgesuchte Bevölkerung bestimmt ${ }^{23}$.

Woraus besteht diese humanitäre Hilfe ? Zunächst macht sie etwas weniger als ein Fünftel der für die internationale Zusammenarbeit der Schweiz aufgebrachten Gesamtbeträge aus, das heisst durchschnittlich rund 250 Millionen Franken. Doch muss diese Zahl genau analysiert werden. Denn sie besteht zum Grossteil aus ordentlichen und ausserordentlichen Beiträgen - zuweilen aus Nahrungsmittelhilfe - an eine Reihe internationaler Organisationen und Nichtregierungsorganisationen. Die humanitäre Hilfe der Schweiz läuft somit in erster Linie über Partnerinstitutionen.

Tabelle Nr. 1: Humanitäre Hilfe des Bundes 1996

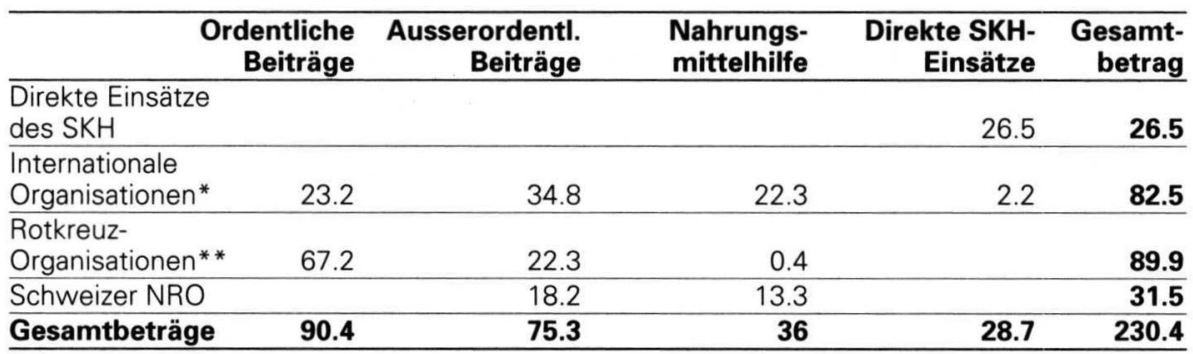

Quelle: Jahresbericht der Entwicklungszusammenarbeit 1996.

* Das Welternährungsprogramm (WEP) und das Hochkommissariat der Vereinten Nationen für Flüchtlinge (UNHCR) erhalten drei Viertel der an die internationalen Organisationen ausgezahlten Beträge.

** Von diesem Betrag erhält das IKRK 83.2 Millionen Franken, der Restbetrag geht an das Schweizerische Rote Kreuz.

Der Blick auf die Zahlen trügt. Er könnte den Eindruck erwecken, dass die Hilfe des Bundes hauptsächlich auf den indirekten Einsatz von Dritten ausgerichtet ist und die direkten Einsätze dabei nur eine Nebenrolle spielen. Dies hiesse, die Bedeutung dieser direkten Einsätze, bei denen die Angehörigen des Schweizerischen Katastrophenhilfekorps (SKH) engagiert werden, herabzusetzen. Diese Einsätze sind in eine Gesamtkonzeption integriert, die aus einer klaren Strategie hervorgeht, welche um vier Ziele - Vorsorge, Rettung, Überleben und Wiederaufbau - gegliedert ist. Das entspricht vier Stadien, von denen jedes unterschiedliche Einsatzformen erfordert: Überwachung, um zu verhüten; Soforthilfe, um zu retten; Wasserversorgung, Nahrung, Unterkünfte und medizinische Betreuung, um das Überleben und die Wiederinstandsetzung der Infrastruktur sicherzustellen, erste Etappe eines Wiederaufbauprozesses, bei dem die Entwicklungszusammenarbeit die Fortsetzung zu übernehmen hat.

Zweiter wichtiger Bereich der Solidaritätspolitik, der nicht unter die Rubrik der humanitären Politik fällt, die Asylpolitik, die sehr lange von der internationalen Flüchtlingspolitik getrennt behandelt wurde, welche ihrerseit unter diese Rubrik fällt. Auch heute bleibt - selbst wenn eine gewisse Zusammenarbeit existiert - noch immer eine Welt zwischen dem Asylbereich und der internationalen Flüchtlingspolitik bestehen. Eine Welt, die allmählich dazu neigt, kleiner zu 
werden $^{24}$. Die Gründe für diese Kluft sind nicht explizit. Sie sind jedoch verständlich. Diese beiden grossen Tätigkeitsbereiche unterstehen zwei verschiedenen Departementen. Bis vor kurzem, das heisst bis ganz zu Ende der 80er Jahre, hat der Flüchtlingsstrom auch kaum die verschiedenen Probleme aufgeworfen, die heute Schlagzeilen in den Medien machen, und hat nicht zu Ausgaben geführt, die von rund 150 Millionen Franken 1986 auf über eine Milliarde Franken 1997 angestiegen sind, und die 1998 die vom Bund für die internationale Zusammenarbeit aufgebrachten Gesamtbeträge übersteigen könnten ${ }^{25}$.

In zehn Jahren hat sich die Situation demnach vollkommen verändert. Seit 1996 übersteigen die humanitären Aufwendungen des Bundes - das heisst die Summe der humanitären Hilfe und der Kosten für die Unterstützung der Flüchtlinge in der Schweiz - seine Aufwendungen für die Entwicklungszusammenarbeit. Für 1998 dürften letztere bei 1'050 Millionen Franken liegen, die den 1'400 Millionen für die humanitären Hilfe gegenüberzustellen sind, die sowohl ausserhalb als auch innerhalb des Landes geleistet wird. In der Tat geht es darum, den Gesamtaufwand und nicht nur die Kosten für die ausserhalb der Schweiz erbrachte Hilfe zu berücksichtigen. Die Schweiz wird auf diese Weise - und sie ist nicht die einzige - immer mehr in einen humanitären Teufelskreis hineingezogen. Je mehr ihre Aufwendungen für den humanitären Bereich zunehmen, desto mehr bleiben die Summen, die sie für ihre Politik der Entwicklungszusammenarbeit einsetzen könnte, auf einen gewissen Niveau stehen oder gehen sogar zurück. Eine gute Entwicklungszusammenarbeit ist nun aber eines der besten Instrumente zur Verhütung humanitärer Krisen. Jede Verringerung der Entwicklungszusammenarbeit läuft somit Gefahr, zu einer Zunahme dieser Krisen und zu ihrer Vertiefung beizutragen.

Tabelle Nr. 2: Internationale Zusammenarbeit des Bundes und Flüchtlingshilfe (1995-1997)

\begin{tabular}{lrrr}
\hline & $\mathbf{1 9 9 5}$ & $\mathbf{1 9 9 6}$ & $\mathbf{1 9 9 7}$ \\
\hline Entwicklungszusammenarbeit & 972.9 & 976.8 & 943.8 \\
\hline Humanitäre Hilfe & 244.8 & 230.4 & 222.1 \\
\hline $\begin{array}{l}\text { Beiträge für die mittel- und } \\
\text { Osteuropäischen Länder }\end{array}$ & 115.7 & 143.3 & 131.1 \\
\hline $\begin{array}{l}\text { Gesamtbetrag I } \\
\text { internationale Zusammenarbeit) }\end{array}$ & $\mathbf{1 3 3 3 . 4}$ & $\mathbf{1 3 5 0 . 5}$ & $\mathbf{1 2 9 7 . 0}$ \\
\hline Flüchtlingshilfe & 811.8 & 927.8 & 1016.5 \\
\hline Gesamtbetrag II & $\mathbf{2 ~ 1 4 5 . 2}$ & $\mathbf{2 2 7 8 . 3}$ & $\mathbf{2 ~ 3 1 4 . 5}$ \\
\hline
\end{tabular}

Quellen: Budgets des BFF von 1986 bis 1998, Bern, Bundesamt für Flüchtlinge, 2.9.1998; Jahresberichte der internationalen Zusammenarbeit 1996 und 1997.

24. Die Anmerkungen von Peter Arbenz sind diesbezüglich sehr aufschlussreich: «Mindestens bis vor der Jugoslawienkrise operierte die Direktion für Entwicklungszusammenarbeit und humanitäre Hilfe (DEH) in der internationalen Flüchtlingspolitik praktisch unabhängig vom Bundesamt für Flüchtlinge (BFF) und unterstützte bei internationalen Flüchtlingskrisen primär die hierfür zuständigen internationalen Organisationen. Zudem führte sie mit ihrem Katastrophenhilfekorps ihre eigenen Projekte und Programme durch. Auch die übrigen für die Aussenpolitik zuständigen Direktionen des EDA betrieben lange Zeit ihre eigene, von der schweizerischen Asylpolitik unabhängige Menschenrechtspolitik, bzw. ihre Politik der internationalen Zusammenarbeit. Als der Bundesrat 1988 einen Koordinator für die internationale Flüchtlingspolitik einstellte, verbesserte sich die interdepartementale Zusammenarbeit zwischen dem EDA und dem EJPD.» Peter Arbenz, Bericht über eine schweizerische Migrationspolitik, Mai 1995, S. 43-44.

25. Die für 1998 veranschlagte Summe beträgt 1'158'083'000.- Franken. Vgl. die Budgets des BFF von 1986 bis 1998 , Bern, Bundesamt für Flüchtlinge, 2.9.1998. 


\section{Graphik Nr. 1: Vergleichende Entwicklung der öffentlichen Hilfe des Bundes und der Aufwendungen des Bundesamtes für Flüchtlinge (1986-1998)}

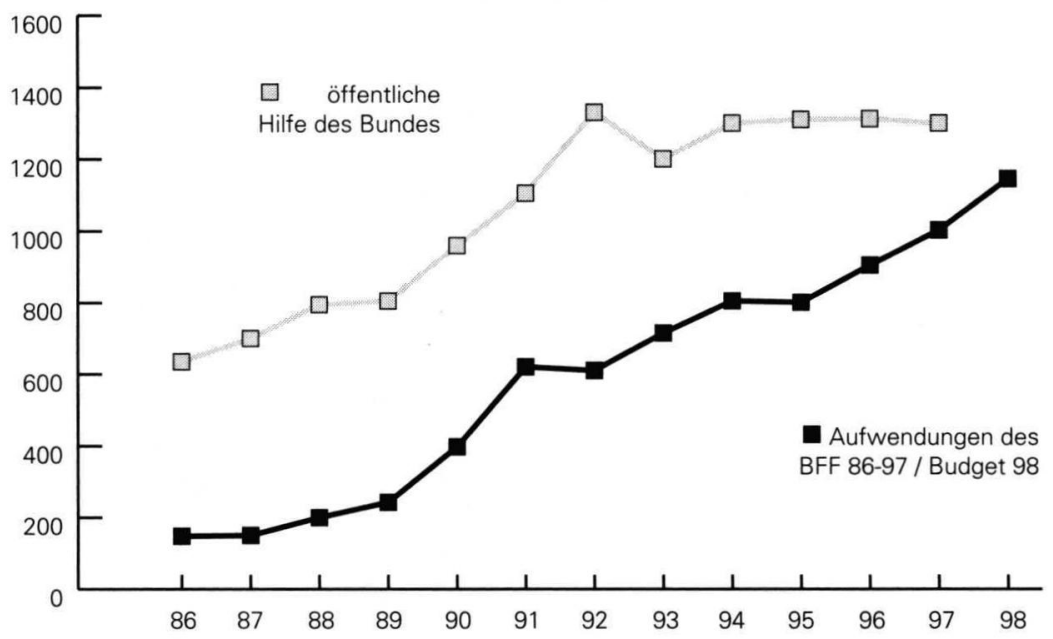

Quellen: Budgets des BFF von 1986 bis 1998, Bern, Bundesamt für Flüchtlinge, 2.9.1998; Jahresberichte der internationalen Zusammenarbeit 1996 und 1997 und andere Quellen.

Grundsätzlich umfasst die internationale Flüchtlingspolitik zunächst eine ganze Reihe Tätigkeiten, die das internationale Flüchtlingsrecht und seine Anwendung sowie allgemein die internationale Flüchtlingspraxis betreffen. Aber von allen Dimensionen der internationalen humanitären Politik der Schweiz weist diese Flüchtlingspolitik ein wesentliches Merkmal auf, das die anderen Dimensionen nicht haben. Sie ist als einzige ganz eng mit einer Problematik verbunden, die zur Innenpolitik gehört, nämlich der Asylpolitik ${ }^{26}$. Daraus ergibt sich die Tatsache, dass die Schweiz in der Flüchtlingspolitik in erster Linie ihre Interessen wahren muss, bevor sie einen Ansatz fördert, dessen Zweck wäre, die Anliegen der humanitären Sache und der Menschenrechte zu fördern.

Dieses Problem stellt sich kaum im Bereich der Menschenrechte, wo die Schweiz, mit einigen seltenen Ausnahmen, nicht auf der Anklagebank sitzt und ihre Praxis nicht zu verteidigen hat. Die Schweiz hat erst seit Anfang der 80er Jahre ausdrücklich eine wirkliche Menschenrechtspolitik, genau gesagt seit 1982, als der Bundesrat seine Politik in seinem Bericht über die schweizerische Menschenrechtspolitik festlegt ${ }^{27}$. Die Botschaft betreffend ein Gesetz über die Entwicklungszusammenarbeit spricht das Problem der Menschenrechte übrigens nicht an. Seither werden die Förderung und Erhaltung der Menschenrechte formell als einer der Bereiche der humanitären Politik angesehen ${ }^{28}$, aber als ein unterschiedlicher Bereich, der auf einer anderen Ebene als die übrigen Komponenten dieser Politik liegt. Zwar «gehen die Konflikte jeder Art, mit deren Opfern die humanitäre Hilfe ständig konfrontiert ist, meistens auch mit Menschenrechtsverletzungen einher $»^{29}$, aber das Wesen der humanitären Hilfe

26. Walter Kälin, «Die schweizerische Flüchtlings- und Asylpolitik», Neues Handbuch der schweizerischen Aussenpolitik, S. 761-775.

27. Bericht des Bundesrates über die schweizerische Menschenrechtspolitik, vom 2. Juni 1982, BB1 134, II, 729.

28. Botschaft über die Weiterführung der internationalen humanitären Hilfe vom 25. Mai 1988, S. 1213.

29. Ibid., S. 1222. 
ist es schliesslich, sich durch einen direkten Einsatz prioritär darum zu bemühen "gefährdetes Leben zu retten und Leiden zu lindern " ${ }^{30}$. Die humanitäre Aktion ist unmittelbar und konkret. Die Aktion zugunsten der Menschenrechte ist allgemeiner. Sie entfaltet den wesentlichen Teil ihrer Wirkungen langfristig und strebt vor allem die Entwicklung der Menschenrechte und die Förderung von Mechanismen an, die den Zweck haben, die Einhaltung dieser Rechte zu kontrollieren. Bisweilen jedoch stimmen die humanitäre Aktion und die Wahrung der Menschenrechte überein. So, wenn es darum geht, Einzelpersonen zu schützen, welche Opfer von Menschenrechtsverletzungen $\operatorname{sind}^{31}$.

Die Förderung und die Entwicklung des humanitären Völkerrechts gehören ebenfalls formell zur humanitären Politik der Eidgenossenschaft. Aber in einer vielleicht ausdrücklicheren und direkteren Art und Weise als im Fall der Menschenrechte. Dies zu allererst aus geschichtlichen Gründen. Die Eidgenossenschaft berief die Konferenz ein, welche im August 1864 die erste Genfer Konvention annimmt, und auf diesen Zeitpunkt geht die Rolle der Schweiz bei der Entwicklung des humanitären Rechts zurück. Die Eidgenossenschaft ist es auch, die alle aufeinanderfolgenden diplomatischen Konferenzen einberufen wird. Zweiter Grund, die Verantwortung der Eidgenossenschaft als Depositarstaat der Genfer Konventionen und ihrer Zusatzprotokolle. Diese Verantwortung legt der Schweiz Verpflichtungen auf. Ihr obliegt es, den Parteien Informationen über die Konventionen zu übermitteln und sie im besonderen von den weiteren Ratifikationen und Beitritten in Kenntnis zu setzen, ganz abgesehen von gewissen Aufgaben, die der Schweiz sowohl von den Abkommen als auch von den Protokollen übertragen werden. Diese Verantwortung scheint die Eidgenossenschaft übrigens - wahrscheinlich mehr, als wenn sie sie nicht übernommen hätte - dazu gebracht zu haben, sich aktiv für die Förderung des humanitären Rechts und seine Weiterentwicklung einzusetzen. Dritter Grund schliesslich, der die Sache betrifft. Das humanitäre Recht steht zweifellos der humanitären Aktion viel näher als die Menschenrechte ${ }^{32}$.

Letzte Komponente der humanitären Politik der Eidgenossenschaft, ihre Unterstützung des IKRK. Eine Unterstützung, die mehrere Formen annimmt und die, wie man sie auch immer bewertet, sehr gewichtig ist. Diese Unterstützung ist übrigens nicht einseitig. Denn der Gedanke des Roten Kreuzes, die Organisationen, die zu seiner Förderung gegründet wurden und die Aktivitäten, die in seinem Namen durchgeführt werden, haben viel zum Image einer «humanitären Schweiz» als Wiege des Roten Kreuzes beigetragen, das der Schweiz zugute kommt und das ihr gleichzeitig zumindest moralische Verpflichtungen auferlegt. «Das Genfer Komitee, liest man in einem Bericht des EPD vom Februar 1944, ist eine internationale Institution, der gegenüber unser Land mehr Pflichten, aber nicht mehr Rechte als irgendein anderer Staat hat ${ }^{33}$. Fünfzig Jahre später behält diese Aussage ihren vollen Wert und bleibt das Fundament der Politik des Bundes, der das Anliegen hat, ständig die besondere Art der Genfer Institution, ihre Unabhängigkeit, ihren nichtstaatlichen, neutralen Charakter und ihre

30. Ibid.

31. Mathias-Charles Krafft und Jean-Daniel Vigny, «La Politique suisse à l'égard des droits de l'homme», Nouveau Manuel de la politique extérieure suisse, Bern, Haupt, 1992, pp. 223-245.

32. Yves Sandoz, «La Suisse et le droit international humanitaire, en particulier les conventions de Genève pour la protection des victimes des conflits armés, Nouveau Manuel de la politique extérieure suisse, pp. 247-263.

33. «Proposition du Département politique au Conseil fédéral» du 14 février 1944, Documents Diplomatiques suisses 1848-1945, Vol. 15 (8.9.1943-8.5.1945). Berne, Benteli Verlag, 1992, p. 226. 
internationale Ausrichtung hervorzuheben. Sowohl das IKRK als auch Bern wollen diese Neutralität jedoch nicht mit der Neutralität der Schweiz verbunden wissen. Genauer gesagt: "Auch wenn sich die Neutralität des IKRK und jene der Schweiz ergänzen, dürfen sie dennoch nicht miteinander verwechselt werden $»^{34}$. Beide wollen, dass das IKRK keineswegs als Instrument der Aussenpolitik der Schweiz und insbesondere seiner humanitären Politik in Erscheinung tritt. Es besteht eine Interessengemeinschaft und eine Übereinstimmung in den Zielsetzungen. Gewisse Verantwortungen sind sogar geteilt und zwingen zu einer oft engen Zusammenarbeit, besonders bei allem, was die Verbreitung und Entwicklung des humanitären Völkerrechts betrifft. Die zwischen Bern und dem Komitee geknüpften Bande sind somit sehr eng, mit dem entschlossenen Willen beider Seiten, alles zu unternehmen, damit das IKRK zu keinem Zeitpunkt diese zur Erfüllung seiner Aufgabe unerlässliche Unabhängigkeit verliert. Und zwar ungeachtet der bedeutenden - insbesondere finanziellen - Unterstützung, die der Bund dem IKRK zukommen lässt. Über 65 Millionen Franken sind im Finanzplan für die Jahre 1998 bis 2000 angesetzt und zur Finanzierung des Sitzbudgets bereitgestellt ${ }^{35}$ und rund 30 Millionen Franken sollten durchschnittlich als Beitrag zum ausserordentlichen Budget der Institution gezahlt werden ${ }^{36}$. Das heisst eine Summe, die insgesamt rund ein Drittel der Aufwendungen des Bundes im Rahmen der humanitären Hilfe ausmacht und die Eidgenossenschaft mit ihrem Beitrag zum IKRK-Budget zum dritten Geber nach den Vereinigten Staaten und der Europäischen Union werden lässt.

Diese rasche Beschreibung der Hauptkomponenten der humanitären Politik gibt zu einigen Bemerkungen Anlass. Ohne jeden Zweifel weist der gewählte Ansatz eine gewisse Kohärenz auf. Eine Reihe von Aktivitäten mit gemeinsamen Nennern zusammengefasst zu haben, geht aus einer erklärlichen Logik hervor. Auch wenn man manchmal den Eindruck hat, dass einige dieser Aktivitäten ganz verschiedenen Bereichen angehören. Eine der konzeptuellen Schwierigkeiten hat ihren Ursprung darin, das man unter ein und derselben Rubrik die humanitäre Politik im engeren Sinne zusammenfassen wollte, jene, die auf die Krisensituationen ausgerichtet ist, wo es darum geht, in äusserster Dringlichkeit zu retten, und die humanitäre Politik im weiteren Sinne, deren Zweck es ist, « den

34. Botschaft des Bundesrates über die Finanzhilfe des Bundes für das Sitzbudget des Internationalen Komitees vom Roten Kreuz (1998-2001) vom 2. Juni 1997, BB1 149, 1997, V, S.74. Diese Frage der Neutralität und der jeweiligen Neutralität des IKRK und der Schweiz ist Gegenstand zahlreicher Debatten gewesen und die diesbezüglichen Meinungen haben sich weiterentwickelt. Der Geschichte halber sei daran erinnert, dass die Neutralität des Sanitätspersonals eines der Grundprinzipien des Rotkreuzgedankens darstellt, auf das sich die Teilnehmer der Konferenz einigen, die vom Genfer Komitee Ende Oktober 1863 einberufen wird. Seltsam ist, dass nicht die Mitglieder des Komitees am meisten an dieser Neutralität hängen, von der Gustave Moynier im übrigen nicht wünscht, dass sie diskutiert wird, sondern diejenigen Teilnehmer, die wegen ihrer Kriegserfahrung feststellen konnten, dass Ärzte und Krankenpfleger als Kombattanten angesehen werden und aus diesem Grunde zur Zielscheibe genommen, verwundet oder getötet werden. Einmal mehr der Beweis dafür, dass der Wert der Neutralität nicht von denen am besten beurteilt wird, die sie zu ihrem Anliegen gemacht haben, sondern von denjenigen, denen sie zugute kommt. Vgl. Pierre Boissier, Histoire du Comité International de la Croix-Rouge, S. 107. Eine andere Debatte, die vor etwa zwanzig Jahren stattfand und in den Rahmen der Reflexionen über den Beitritt der Schweiz zur Organisation der Vereinten Nationen (UNO) fällt. Die Auswirkungen eines UNO-Beitritts auf die Tätigkeit des IKRK sind Gegenstand einer eingehenden Diskussion in der Beratenden Kommission für das Verhältnis der Schweiz zur UNO. Das IKRK wird aufgefordert, seine Meinung abzugeben. Dies geschieht in einem Memorandum, in dem festgestellt wird, dass «die Aktion des IKRK im wesentlichen auf der Neutralität der Schweiz beruht, ob in Kriegen, Bürgerkriegen oder bei inneren Unruhen... Dank dieser Neutralität haben die kriegsführenden Parteien eine zusätzliche Garantie für die Unabhängigkeit und die Unparteilichkeit des IKRK». Eine Meinung, der sich die Kommission anschliesst. Vgl. Rapport de la Commission consultative de la Suisse avec l'ONU au Conseil fédéral du 20 août 1975, p. 55.

35. Ibid.

36. Botschaft über die Weiterführung der internationalen humanitären Hilfe vom 20. November 1996. 
Menschen, seine Existenz, seine Würde und Freiheit zu schützen ${ }^{37}$. Eines der edelsten Ziele, das völlig gerechtfertigt ist, das aber operationelle Probleme ganz anderer Art aufwirft als die humanitäre Politik im engeren Sinne.

Ein weiteres Problem, die ganz natürliche Verwechslung, die man zwischen humanitärer Politik und humanitärer Hilfe zu machen neigt. Auch hier steht man vor einem konzeptuellen Problem, das sich aus der Tatsache ergibt, dass man einfach grosse Komponenten nebeneinandergesetzt hat, ohne sich zu viele Fragen zu stellen - nicht über das, was sie gemeinsam haben, was auf der Hand liegt - sondern über die Art und Weise, sie miteinander zu verbinden, ein Ganzes daraus zu machen, das in operationeller Hinsicht sinnvoll wird.

Dritte Bemerkung, die Tatsache, dass streng genommen, die Entwicklungszusammenarbeit und die Asylpolitik aus der humanitären Politik im weiteren Sinne herausgehalten werden, erstaunt. Der Logik nach sollten sie im Kern der Asylpolitik sein, da es um «den Menschen, seine Existenz, seine Würde und Freiheit» geht. Wie lässt sich diese Tatsache erklären? Eine Antwort wurde weiter oben in Bezug auf die Asylpolitik angedeutet, die zugleich ein innenpolitisches Problem ist und einem anderen Departement als dem eidgenössischen Departement für auswärtige Angelegenheiten (EDA) untersteht. Ganz abgesehen davon, dass das Asylproblem erst in den letzten zehn Jahren explosionsartig angewachsen ist. Und dennoch gibt es nichts humanitäreres als die Flüchtlings- und Asylproblematik? Was die Entwicklungszusammenarbeit betrifft, abgesehen von der Zusammenarbeit mit Osteuropa, die man Tendenz hat, zu vergessen, liegt die Erklärung zweifellos mehr bei der Geschichte, beim institutionellen Aufbau, bei praktischen Fragen betreffend die Finanzierungsanträge, die leichter der Reihe nach zu bearbeiten sind, als bei der Logik.

Diese konzeptuellen Probleme wären ohne grosse Bedeutung, wenn sie keine praktische Folgen hätten. Konzepte sind nicht unschuldig. Sie wirken sich auf die Strukturen aus, auf die Art, zu denken, zu reden und zu handeln, auf die Weise, wie Institutionen und Menschen zusammenarbeiten oder sich ignorieren. Was sich seinerseits auf die Kohärenz der Politiken, ihre Umsetzung und ihre Effizienz auswirkt. Alles Dinge übrigens, deren sich die Bundesverwaltung bewusst ist und an denen sie arbeitet.

7um ABSCHLuSs dieses Jahrhunderts ist die humanitäre Sache nicht mehr das,

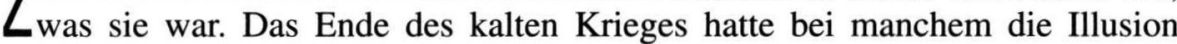
geweckt, das Ende der Geschichte sei gekommen. Nun erweist sich jedoch, dass die Geschichte kein Ende hat. Der Hoffnung auf bessere Zeiten ist ein Bewusstwerden gefolgt, dass die Herausforderungen, mit denen die Menscheit konfrontiert ist, nur noch an Umfang und Komplexität zunehmen. Allein im humanitären Bereich im engeren Sinne häufen sich Naturkatastrophen und technische Katastrophen. Das gleiche gilt für die Konflikte aller Art, die keinen Kontinent verschonen.

Aber die humanitäre Sache ist auch nicht mehr, was sie war, weil sie zum allgemeinen Gerangel, zum Gegenstand wichtiger auf dem Spiel stehender Entscheidungen, zum Objekt von Kontroversen, Rivalitäten und Kräften gewor- 
den ist. Sie hat es nicht vermeiden können, sich oft zu einer Geisel der Politik zu verwandeln, zum Alibi und Schutzschirm von Regierungen, die hinter der humanitären Sache sowohl ihre Kleinmütigkeit verbergen, als auch sich Praktiken hingeben können, die dazu beitragen, Öl ins Feuer zu giessen. Auch die Opfer werden als Geiseln genommen. Einige sind Opfer der Sache, für die man eintritt. Und dann gibt es andere Opfer, für die keine Sache eintritt, die ihre Tragödie fern von den Medien in Vergessenheit erleben. Schliesslich zieht die humanitäre Sache Milliarden an sich und ist ganz natürlich oft zum «Geschäft» geworden, wie stark auch immer der Glaube und der Idealismus jener ist, die sich für die humanitäre Sache, besonders vor Ort engagieren. Das Phänomen hat übrigens nicht erst mit dem Ende des Kalten Krieges begonnen. Es geht auf den Biafrakrieg zurück und hat sich immer weiter verbreitet ${ }^{38}$.

In diesem neuen Kontext hat die Bundesverwaltung eine Reflexion eingeleitet, die in der Botschaft des Bundesrates über die Weiterführung der internationalen humanitären Hilfe vom 20. November 1996 gut wiedergegeben ist. Diese Reflexion wurde im Rahmen einer Arbeitsgruppe über die humanitäre Politik der Schweiz fortgeführt, die von der Politischen Abteilung IV des EDA (Menschenrechts- und humanitäre Politik) eingesetzt wurde und deren Schlussbericht in Kürze erhältlich sein dürfte. Parallel dazu hat man im gesamten Verlauf der 90er Jahre Umstrukturierungen und Änderungen in der Praxis erlebt, die alle zum Ziel hatten, der humanitären Politik mehr Kohärenz und Effizienz zu verleihen. Die Gründung der Politischen Abteilung IV ist hierfür ein Beispiel ${ }^{39}$.

Ausgangspunkt der Reflexion der Verwaltung ist der Bericht über die Aussenpolitik der Schweiz in den 90er Jahren vom November 1993 und die fünf aussenpolitischen Ziele, um die der Bericht sich gliedert:

๑ Wahrung und Förderung von Sicherheit und Frieden,

- Förderung von Menschenrechten, Demokratie und Rechtsstaat,

- Förderung der Wohlfahrt,

- Abbau sozialer Gegensätze,

- Schutz der natürlichen Lebensgrundlagen.

Die für die humanitäre Hilfe des Bundes Verantwortlichen bringen diese Hilfe absichtlich in den vom Bericht festgelegten Rahmen ein. Sie integrieren ihre Definition der humanitären Aktion in ein Ganzes, das nicht nur die Aussenpolitik, sondern auch die Sicherheitspolitik, die Entwicklungspolitik und die Migrationspolitik umfasst. Sie folgen dem Begriff der Komplementarität der Politiken, der Instrumente und der Aktion. Sie denken in Nischen. Sie erfassen genau, worin andere aus der Sicht der humanitären Aktion eine zentrale Rolle

38. Die humanitäre Sache ist in Mode. Sie ist Gegenstand zahlreicher Bücher, von denen einige das hier Gesagte gut veranschaulichen. Vgl. Mario Bettati, Le droit d'ingérence. Mutation de l'ordre international, Paris, Editions Odile Jacob, 1996. Oder auch Bernard Debré, L'illusion humanitaire. Paris, Plon, 1997.

39. Nachdem Peter Arbenz im obenerwähnten Bericht die Einsetzung des Postens eines Koordinators für internationale Flüchtlingspolitik 1988 angesprochen hat, fährt er fort: «Die Hauptaufgaben des Koordinators bestehen darin, das EDA für die Probleme des BFF bei der Anwendung der Asylpolitik zu sensibilisieren und das EDA dazu zu bringen, dem BFF seine diplomatischen Ressourcen zur Verfügung zu stellen. Dies war vor allem in einer Phase der Fall, als sich die internationalen Fora für Fragen der Asyl- und Migrationspolitik ständig vermehrten. Diese Zusammenarbeit hat den Abschluss verschiedener bilateraler Abkommen über die Rücknahme von Personen ermöglicht und den Dialog mit den europäischen Partnern im Interesse der Wirksamkeit und Glaubwürdigkeit der schweizerischen Asylpolitik erlaubt.

Mit der Reorganisation des EDA, die vom Bundesrat Ende 1994 gebilligt wurde, ist der Koordinator von jetzt an der neugegründeten Politischen Direktion IV zugeordnet. Diese kümmert sich, mit einem aufgestockten Personalbestand, um die humanitäre Politik, die Menschenrechtspolitik und die internationale Flüchtlingspolitik.» Bericht über eine Migrationspolitik, p. 48 (fr.). 
zu spielen imstande sind, insbesondere was die Verhütung von Konflikten und Katastrophen betrifft. Präventivdiplomatie und gute Dienste, Zusammenarbeit im Bereich der Überprüfung, der Abrüstung und der Kontrolle des Waffenhandels, Armutsbekämpfung, Einsatz für Demokratie und Rechtsstaatlichkeit, für die Achtung der Menschenrechte und den Schutz von Minderheiten, Bekämpfung der Ursachen der Migrationsbewegungen, alles wird aus der Perspektive einer humanitären Aktion berücksichtigt, bei der es zugleich darum geht, die Aktion zu erleichtern, indem man durch die Prävention vermeidet, sie überhaupt einsetzen zu müssen, oder auch, indem man sie unterstützt, wenn sie erforderlich wird. Dies ist ein wichtiger Wendepunkt im Denken. Auf den sektoriellen Ansatz folgt eine Vision, die aus einem Ganzen hervorgeht, der Politik des Staates, zwar zu allererst seiner Aussenpolitik, aber auch seiner Innenpolitik, insofern als beide untrennbar sind ${ }^{40}$.

Der Bericht der Arbeitsgruppe «Humanitäre Politik», der sich in der Ausarbeitung befindet, geht noch weiter. Er ist bestrebt, die humanitäre Politik in kohärenter Weise zu konzeptualisieren. Er legt vier Ziele fest: Schutz des Lebens und der menschlichen Würde in akuten Krisensituationen, Förderung humanitärer Werte, Prävention und Wiederaufbau. Anschliessend beschreibt er in diesem Rahmen die Funktion der Hauptkomponenten der humanitären Politik: Entwicklung und Förderung des humanitären Rechts und der Menschenrechte, Aktivitäten der Hilfe und Zusammenarbeit, friedensfördernde Aktivitäten.

Humanitäres Recht und Menschenrechte sind dadurch miteinander verbunden, bleiben aber gleichzeitig unterschiedlich. Dies macht Sinn im Hinblick auf die laufenden Bemühungen, «Mindeststandards der Menschlichkeit» zu definieren. Das gleiche gilt für die humanitäre Hilfe und die Entwicklungszusammenarbeit, die unter ein und derselben Rubrik zusammengefasst sind. Schliesslich der dritte Bereich, die friedensfördernden Aktivitäten in der doppelten Form von Beteiligungen an Friedens- und Vermittlungsoperationen oder, um in der Kontinuität der Aussenpolitik der Schweiz zu bleiben ${ }^{41}$, von guten Diensten, aber erneuerten guten Diensten, die dem Zeitgeist, dem Kontext und der Praxis der Zeit angepasst sind.

Was überraschen mag, ist die Tatsache, dass die gesamte Flüchtlings-, Migrations- und Asylproblematik nicht in dieses Konzept integriert ist. Zweifelsohne erklärt sich dies aus guten Gründen. Jedoch scheint es merkwürdig, dass der Bereich der humanitären Politik, der die grössten Probleme verursacht und Summen verschlingt, die den Gesamtaufwendungen im Rahmen der internationalen Zusammenarbeit der Schweiz entsprechen und diese vielleicht bald übersteigen, aus einem Konzept herausgelassen wird, das zu Recht alle Komponenten berücksichtigen will, die einen humanitären Aspekt aufweisen. 1998 wurde eine Milliarde Franken, das heisst das Doppelte des für bilaterale technische Zusammenarbeit bewilligten Betrags, zur Finanzierung des Asyls von 140'000 Personen aufgebracht, die sich aus dem einen oder anderen Grunde per Ende August dieses Jahres in der Schweiz aufhielten. Dies ist ohne Zweifel die gewaltigste humanitäre Herausforderung, welche die Schweiz heute lösen muss

40. Botschaft über die Weiterführung der internationalen humanitären Hilfe vom 20. November 1996.

41. Vgl. das klassische Werk über gute Dienste von Raymond R. Probst, "Good Offices», in International Relations in the Light of Swiss Practice and Experience. Dordrecht, Martinus Nijhoff, 1988. 
und die nur überwunden werden kann, indem man damit beginnt, sie in ein Gesamtkonzept einzugliedern ${ }^{42}$.

Der humanitäre Bereich in der Aussenpolitik der Schweiz ist heute auf halbem Wege angelangt. Eine Vision, Ziele und eine Strategie werden derzeit festgelegt. Dies ist eine wesentliche Etappe. Das Schwerste bleibt noch zu tun, nämlich die Umsetzung dieser Strategie. Was die einen und die anderen ganz selbstverständlich zwingen sollte, anders zu arbeiten. In diesem Zusammenhang wird oft über Koordination gesprochen. Die Koordination ist aber kein Allheilmittel. Sie wird nur selten von denen akzeptiert, die koordiniert werden. Was zu fördern ist, ist vielmehr die Dezentralisierung der Verantwortungen, der Informationsfluss, der Dialog und die Zusammenarbeit, und zwar im Rahmen gemeinsamer Projekte, die allen gehören. Auch hier bewegt sich die Praxis, zwar langsam, jedoch ist dies für die vor sich gehenden Veränderungen aufschlussreich. Die gemeinsame Reise der Direktoren der DEZA und des BFF in das frühere Jugoslawien ist ein gutes Beispiel dafür. Die Herausgabe der Leitlinien über Förderung der Menschenrechte und Entwicklungszusammenarbeit ist ein weiteres Beispiel $^{43}$.

An den Ufern des Kongo mehren sich die humanitären Herausforderungen. Bald wird es vielleicht an Nahrung mangeln. Und der Krieg dort unten, am anderen Ende des Landes geht weiter, ausser Sicht der Medien, die ferngehalten werden. Wie weit der Weg von Bern nach Kinshasa doch ist, von den Konzepten und Politiken zur alltäglichen Realität von Millionen Menschen, die nichts weiter wünschen, als ein «normales» Leben zu führen, und die unaufhörlich von der Geschichte zermalmt werden.

42. Die Schwierigkeit der Aufgabe wird gut herausgestellt in Marcus Cox, Strategic Approaches to International Intervention in Bosnia and Herzegovina, Genf, Centre for Applied Studies in International Negotiations (CASIN), 1998. 43. Förderung der Menschenrechte und Entwicklungszusammenarbeit. Leitlinien, DEZA, 1997. 